\title{
Distinguishing fingerprints of hyperchaotic and stochastic dynamics in optical chaos from a delayed optoelectronic oscillator
}

\author{
Miguel C. Soriano, ${ }^{1, *}$ Luciano Zunino, ${ }^{2,3}$ Laurent Larger, ${ }^{4}$ Ingo Fischer, ${ }^{1}$ and Claudio R. Mirasso ${ }^{1}$ \\ ${ }^{1}$ Instituto de Física Interdisciplinar y Sistemas Complejos (IFISC) CSIC-UIB, \\ Campus Universitat de les Illes Balears, E-07122 Palma de Mallorca, Spain \\ ${ }^{2}$ Centro de Investigaciones Ópticas (CONICET La Plata-CIC), C.C. 3, 1897 Gonnet, Argentina \\ ${ }^{3}$ Departamento de Ciencias Básicas, Facultad de Ingeniería, Universidad Nacional de La Plata (UNLP), \\ 1900 La Plata, Argentina \\ ${ }^{4}$ Institut FEMTO-ST, CNRS UMR 6174, Université de Franche-Comté, Besançon 25030 Cedex, France \\ *Corresponding author: miguel@ifisc.uib-csic.es
}

Compiled May 11, 2011

\begin{abstract}
In the dynamics of optical systems one commonly needs to cope with the problem of coexisting deterministic and stochastic components. The separation of these components is an important although difficult task. Often the time scales at which determinism and noise dominate the system's dynamics differ. In this letter we propose to use information-theory-derived quantifiers, more precisely permutation entropy and statistical complexity, to distinguish between the two behaviors. Based on experiments of a paradigmatic optoelectronic oscillator we demonstrate that the time scales at which deterministic or noisy behavior dominate can be identified. Supporting numerical simulations prove the accuracy of this identification. (c) 2011 Optical Society of America OCIS codes: $140.1540,190.3100,250.5960,000.5490$.
\end{abstract}

Delayed coupling phenomena play an important role in optical systems, including semiconductor lasers with feedback [1], delay-coupled lasers [2], and optoelectronic oscillators [3]. In particular, the latter have proven to be practical benchmark systems to study delay dynamics $[3,4]$. Moreover, these oscillators have turned out to be versatile systems for novel applications such as chaos communications [5] or generation of ultra-high spectral purity microwaves [6]. The main dynamical features of this test-bed system are well documented and characterized, both from the theoretical and the experimental point of view [7].

Experimental realizations of the optoelectronic oscillator are usually affected by an unpredictable stochastic component. In particular, when the dynamical system is driven into the hyperchaotic regime, it can be hard to distinguish between the deterministic chaotic dynamics and the stochastic component when they coexist. To distinguish between these two components we propose, in this letter, to use quantifiers derived from information theory. In particular, permutation entropy and statistical complexity [8] are good candidates for this task. They have already shown to be successful in identifying the internal structures of time series originated from delay systems $[9,10]$.

To compute these quantifiers, we analyze the time series of the system's dynamics and from them construct a probability distribution of their amplitudes. We choose the Bandt and Pompe method due to its simplicity and effectiveness [11]. Band and Pompe consider the order of neighboring values, by comparing their amplitude values, rather than partitioning the amplitude into different levels. This avoids amplitude threshold sensitivity dependences. The probability distribution of the generated or- dinal pattern for a given time series can be established once an embedding dimension $D$ and an embedding delay time $\tau$ are chosen. The embedding dimension $D$ refers to the number of symbols that forms the ordinal pattern. The embedding delay $\tau$ is the time separation between symbols which is directly related to the sampling time of the time series (see refs. [9-11] for a detailed derivation and description of the quantifiers).

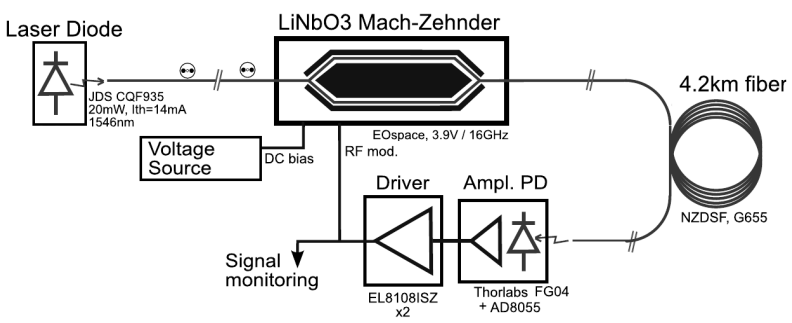

Fig. 1. The optoelectronic oscillator is composed of a semiconductor laser diode feeding a Mach-Zehnder modulator that performs a sine squared nonlinear transformation, an optical delay line, and an optoelectronic feedback for intensity detection, linear filtering, and amplification. This feedback serves as the drive of the MZ modulator, closing the delay loop (delay time $\sim 21 \mu \mathrm{s}$ ).

Our experimental implementation is depicted in Figure 1. This optoelectronic oscillator generates intensity pulsations which are typical for the Ikeda scenario [3, 7], including a period doubling route to chaos. Figure 2 shows the power spectrum of the oscillator output once the system is driven into the hyperchaotic regime. As it can be seen in Fig. 2, relevant spectral contributions can be estimated up to $\sim 6 \mathrm{MHz}$. According to the NyquistShannon criterion, a sampling rate of $f_{N S}=12$ MSam- 
ples/s would be sufficient. Nevertheless, the time series have been acquired with a sampling rate of $f_{s}=500$ MSamples/s and a resolution of 8 bits. As will be shown later, this oversampling is helpful for the noise and determinism identification.

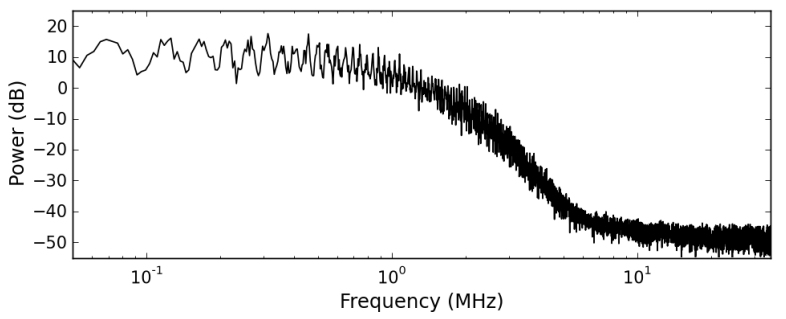

Fig. 2. Power spectrum of the experimentally recorded time-traces of the delayed optoelectronic oscillator operating in the chaotic regime.

Figure 3 illustrates the results of the permutation entropy $(H)$ and the permutation statistical complexity $(C)$ analysis as functions of embedding delay $\tau$ when the system operates in the chaotic regime. Initially, the value of $H$ decreases for increasing $\tau$ in the range $\tau=2 . .40 \mathrm{~ns}$. It reaches a minimum at $\tau=40 \mathrm{~ns}$, and increases for longer $\tau$. On the contrary, the value of $C$ increases for increasing $\tau$ in the range $\tau=2 . .80 \mathrm{~ns}$, reaches a maximum at $\tau=80 \mathrm{~ns}$, and decreases for longer $\tau$. Previous works have shown that the correlations present in deterministic chaotic systems typically yield intermediate values of $H$ ranging from 0.45 to 0.75 and values of $C$ near the maximum, which is $C=0.5$ for $D=6$ [8]. This means that correlated dynamics is found to dominate in the range $\tau=14 . .120 \mathrm{~ns}$ in our experimental realization. Large values of $H$ and small values of $C$ correspond to uncorrelated dynamics $(\tau=2 . .12$ and $122 . .400 \mathrm{~ns})$. The boundary between correlated and uncorrelated dynamics is approximately given by the position of the two extrema in Fig. 3. Interestingly, the embedding delay at which the maximum of $C$ is found, $\tau=80 \mathrm{~ns}$, coincides with the sampling rate obtained with the Nyquist criterion $\left(\tau^{-1} \sim f_{N S}\right)$, i.e. this complexity measure is an alternative to estimate the minimal required sampling time of a chaotic time trace. A similar result has recently been found when analyzing the dynamics of a chaotic semiconductor laser with delayed optical feedback [9].

In the following, we present the results in the entropycomplexity $(H-C)$ plane, as it helps to interpret the quantifiers in an intuitive manner. The $H-C$ plane was introduced in [8] to distinguish between the deterministic chaotic and stochastic nature of a time series. The inset of Fig. 3 is a representation of the pair entropycomplexity at each embedding delay. The value of the quantifiers for $\tau=2 \mathrm{~ns}$ is found at the bottom right corner of the $H-C$ plane. As the embedding delay increases in a clockwise manner, the quantifiers reach an extreme value at the left side and move back to the bottom right corner of the $H-C$ plane. In general, stable or periodic dynamics appear close to the left bottom corner

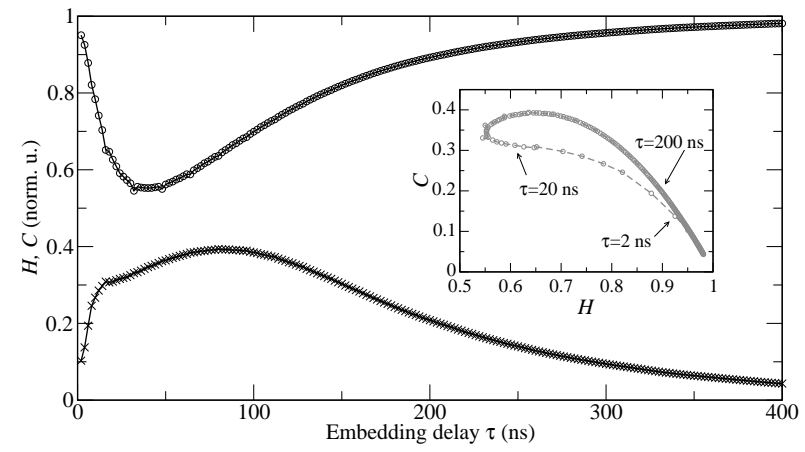

Fig. 3. Permutation entropy ( $H$, circles) and permutation statistical complexity ( $C$, crosses) for an experimental time series recorded in the chaotic regime as functions of $\tau$. The inset represents the pair entropy-complexity at each embedding delay [8]. Embedding dimension $D=6$, and sampling rate $500 \mathrm{MSamples/s}$ are used.

of the plane ( $H$ and $C$ close to zero); correlated dynamics are located in the top center of the plane (intermediate $H$ and $C$ values), while uncorrelated dynamics are found at the bottom right corner of the plane ( $H$ close to one and $C$ close to zero).

The values of the quantifiers as functions of $\tau$ in the inset of Fig. 3 tell us that the system is uncorrelated for small embedding delays, it becomes correlated for an intermediate range of $\tau$, and is again uncorrelated for longer $\tau$. However, with this information alone, it is not possible to identify determinism or stochasticity. Since the bandwidth of the experimental system is narrower than the one of the detection apparatus, we benefit from the oversampling to smooth the original time series. We perform a simple moving average using different window lengths. We take the average value of the points over the respective windows before moving one sample ahead to repeat the procedure. This is performed for the full time series. Figure 4(a) shows the value of the quantifiers in the $H-C$ plane for different lengths of the moving average. It is clear that the position of the quantifiers in the plane is modified when the original time series is smoothed. In particular, the positions of the quantifiers for small embedding delays move gradually from the right hand corner to the left hand corner of the $H-C$ plane for larger lengths of the moving average.

To gain insight into the obtained experimental results we performed numerical simulations of the system. The advantage of using model equations is that it allows us to treat independently the deterministic and stochastic contributions. The deterministic version of the optoelectronic oscillator with delay feedback is described by [12]:

$$
\frac{d x\left(t^{\prime}\right)}{d t^{\prime}}=-x\left(t^{\prime}\right)+\beta \sin ^{2}\left[x\left(t^{\prime}-T\right)+\Phi\right],
$$

where $t^{\prime}$ is the time in normalized units $\left(t^{\prime}=t /(240 \mathrm{~ns})\right)$, $\beta$ is the feedback strength, $T$ is the delay time and $\Phi$ is the phase. The parameters have been rescaled to match the experimental conditions. 


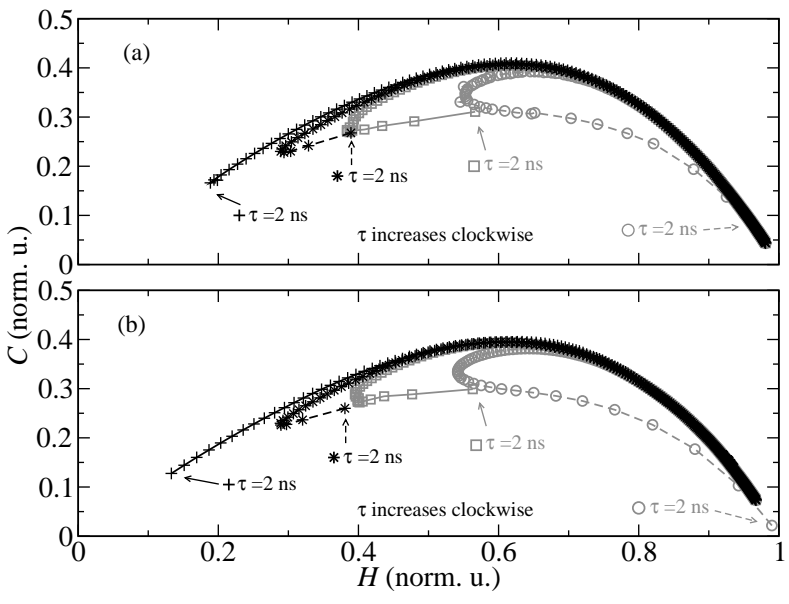

Fig. 4. Values of the permutation entropy and statistical complexity as functions of the embedding delay in the $H-C$ plane for (a) original experimental time series [circles] and corresponding moving averages of different lengths [squares correspond to $20 \mathrm{~ns}$, stars to 40 $\mathrm{ns}$, and crosses to $160 \mathrm{~ns}$ length], and (b) numerical time series without noise [crosses], with added noise [circles] and corresponding moving averages of different lengths [squares correspond to $20 \mathrm{~ns}$ and stars to $40 \mathrm{~ns}$ length]. Parameters: $\beta=3.6, T=87.2, \Phi=0.85$ rad.

For the numerical analysis, we have chosen the parameters such that the system displays chaotic oscillations. Crosses in Figure 4(b) correspond to the permutation quantifiers in the $H-C$ plane for the deterministic system described by Eq. (1). The position of the quantifiers in the $H-C$ plane as function of the embedding delay moves from the bottom-left corner towards the middletop as the embedding delay increases. The final position of the quantifiers lies at the bottom-right corner. We interpret these results as follows, the deterministic chaotic dynamics is (i) oversampled at low values of the embedding delay resembling a pseudo-periodic behavior, (ii) captured at intermediate values of the embedding delay that is near the internal time scale of the system, and (iii) undersampled at large values of the embedding delay resembling a noisy behavior. Qualitatively similar results are found for other values of $\beta$ and $\Phi$, as long as the system stays in the chaotic regime.

The representation of the quantifiers in the $H-C$ plane for the deterministic system (crosses in Fig. 4(b)) and the experimental system with a moving average of 160 ns (crosses in Fig. 4(a)) are very similar to each other. Therefore, we conclude that the uncorrelated component of the experimental time traces at large embedding delays has a deterministic origin. It is a consequence of undersampling the chaotic dynamics rather than originating from a stochastic process.

We can also identify the effect of adding a stochastic component to the system described in Eq. (1) by simply adding a Gaussian white noise to the numerical time series. We chose a noise of zero mean and a standard devi- ation of 0.03 times the standard deviation of the original time series, being compatible with an 8-bits resolution in the acquisition. The circles in Figure 4(b) correspond to this case. From the comparison between experimental and numerical results with moving averages of different lengths we conclude that the uncorrelated component of the experimental time traces at small embedding delays (high frequency range) has a stochastic origin, which is mainly originating from the digitization of the oscilloscope. We have checked that other noise sources can be neglected.

In conclusion, we demonstrated that the method, based on the estimation of permutation entropy and statistical complexity, is able to distinguish between deterministic and stochastic components present in the chaotic dynamics of an optoelectronic oscillator with delayed feedback. The characterization of the quantifiers as functions of the embedding delay reveals the scales at which one or the other component dominates. In particular, characteristic time scales present in the system dynamics can be detected through the presence of clear extrema of the quantifiers. The method proposed here can also be useful in other practical situations, where the deterministic and stochastic components are more subtly distributed in the Fourier spectrum than in the reported experiments. The proposed method could thus be viewed as a spectral correlation distribution of any signal which would consist of a complex contribution of noise and deterministic motions. The size of the embedding delay is defining the inverse of the Fourier frequency for which the deterministic or stochastic contribution is to be evaluated.

\section{References}

1. R. Lang, K. Kobayashi, IEEE J. Quantum Electron. 16, 347-355 (1980).

2. T. Heil, I. Fischer, W. Elsässer, J. Mulet, and C.R. Mirasso, Phys. Rev. Lett. 86, 795-798 (2001).

3. L. Larger, J. M. Dudley, Nature 465, 41-42 (2010)

4. K. E. Callan, L. Illing, Z. Gao, D. J. Gauthier, and E. Schöll, Phys. Rev. Lett. 104, 113901 (2010).

5. A. Argyris, D. Syvridis, L. Larger, V. Annovazzi-Lodi, P. Colet, I. Fischer, J. García-Ojalvo, C. R. Mirasso, L. Pesquera, and K. A. Shore, Nature 438, 343-346 (2005).

6. Y. K. Chembo, L. Larger, H. Tavernier, R. Bendoula, E. Rubiola, and P. Colet, Opt. Lett. 32, 2571-2573 (2007).

7. M. Peil, M. Jacquot, Y. C. Kouomou, L. Larger, and T. Erneux, Phys. Rev. E 79, 026208 (2009).

8. O. A. Rosso, H. A. Larrondo, M. T. Martin, A. Plastino, and M. A. Fuentes, Phys. Rev. Lett. 99, 154102 (2007).

9. M. C. Soriano, L. Zunino, O. A. Rosso, I. Fischer, and C. R. Mirasso, IEEE J. Quantum Electron. 47, 252-261 (2011).

10. L. Zunino, M. C. Soriano, I. Fischer, O. A. Rosso, and C. R. Mirasso, Phys. Rev. E 82, 046212 (2010).

11. C. Bandt and B. Pompe, Phys. Rev. Lett. 88, 174102 (2002).

12. K. Ikeda, Opt. Comm., 30, 257-261 (1979). 